\title{
Snail levels control the migration mechanism of mesenchymal tumor cells
}

\author{
CRISTINA BELGIOVINE ${ }^{1}$, GIULIO CHIESA ${ }^{1}$, ILARIA CHIODI ${ }^{1}$, ROBERTA FRAPOLLI ${ }^{2}$, \\ KATIUSCIA BONEZZI $^{3}$, GIULIA TARABOLETTI ${ }^{3}$, MAURIZIO D'INCALCI ${ }^{2}$ and CHIARA MONDELLO ${ }^{1}$ \\ ${ }^{1}$ Institute of Molecular Genetics, National Research Council, I-27100 Pavia; \\ ${ }^{2}$ Department of Oncology, Mario Negri Institute for Pharmacological Research, I-20156 Milan; \\ ${ }^{3}$ Department of Oncology, Mario Negri Institute for Pharmacological Research, I-24126 Bergamo, Italy
}

Received September 8, 2015; Accepted May 23, 2016

DOI: $10.3892 / 01.2016 .4642$

\begin{abstract}
Cancer cells use two major types of movement: Mesenchymal, which is typical of cells of mesenchymal origin and depends on matrix metalloproteinase (MMP) activity, and amoeboid, which is characteristic of cells with a rounded shape and relies on the activity of Rho-associated kinase (ROCK). The present authors previously demonstrated that, during neoplastic transformation, telomerase-immortalized human fibroblasts (cen3tel cells) acquired a ROCK-dependent/MMP independent mechanism of invasion, mediated by the downregulation of the ROCK cellular inhibitor Round (Rnd)3/RhoE. In the present study, cen3tel transformation was also demonstrated to be paralleled by downregulation of Snail, a major determinant of the mesenchymal movement. To test whether Snail levels could determine the type of movement adopted by mesenchymal tumor cells, Snail was ectopically expressed in tumorigenic cells. It was observed that ectopic Snail did not increase the levels of typical mesenchymal markers, but induced cells to adopt an MMP-dependent mechanism of invasion. In cells expressing ectopic Snail, invasion became sensitive to the MMP inhibitor Ro 28-2653 and insensitive to the ROCK inhibitor Y27632, suggesting that, once induced by Snail, the mesenchymal movement prevails over the amoeboid one. Snail-expressing cells had a more aggressive behavior in vivo, and exhibited increased tumor growth rate and metastatic ability. These results confirm the high plasticity of cancer cells, which can adopt different types of movement
\end{abstract}

Correspondence to: Dr Chiara Mondello, Institute of Molecular Genetics, National Research Council, Via Abbiategrasso 207, I-27100 Pavia, Italy

E-mail: mondello@igm.cnr.it

Abbreviations: MMP, matrix metalloproteinase; ROCK, Rho-associated kinase; EMT, epithelial-mesenchymal transition; MAT, mesenchymal-amoeboid transition; Rnd, Round; PD, population density.

Key words: Snail, Rnd3/RhoE, MMP, ROCK, amoeboid movement, mesenchymal movement in response to changes in the expression of specific genes. Furthermore, the present findings indicate that Rnd 3 and Snail are possible regulators of the type of invasion mechanism adopted by mesenchymal tumor cells.

\section{Introduction}

Cancer cells can adopt two main migration mechanisms, the mesenchymal movement and the amoeboid one, mainly depending on cellular morphology, cytoskeletal organization, cell-extracellular matrix (ECM) interactions and ECM degradation $(1,2)$. The mesenchymal movement is generally used by cells of mesenchymal origin with an elongated morphology and actin organized in stress fibres, and requires the activation of the Rac signaling pathway and ECM-degrading proteases such as matrix metalloproteinases (MMPs) (1). Cells using the amoeboid movement mostly have a round shape and cortical actin bundles. In these cells, actomyosin contraction, which is driven by myosin phosphorylation by Rho-associated kinase (ROCK), is the principal propulsive force, which allows the cells to squeeze through the ECM (3).

In tumor cells of epithelial origin, the shift to the mesenchymal phenotype through the epithelial-mesenchymal transition (EMT) process is associated with the acquisition of the mesenchymal movement, together with high migration and metastatic capacities (4). The EMT can be driven by the expression of different transcription factors, including Snail (which is encoded by the Snail gene) (5). Snail activation is associated with E-cadherin downregulation, adherent junction destabilization, cellular polarization and increased MMP expression (5).

By contrast, the adoption of the amoeboid movement has been described in tumor cells of mesenchymal origin upon inhibition of MMP activity or integrin function (6,7). In addition, variations in $\mathrm{p} 53, \mathrm{p} 27$ or ephrin type-A receptor 2 expression have been reported to lead to a mesenchymal-amoeboid transition (MAT) in sarcoma and melanoma cells (8-10).

The present authors previously reported the occurrence of spontaneous MAT in human telomerase-immortalized fibroblasts, named cen3tel, which underwent malignant transformation during in vitro propagation (11). During the acquisition of the tumorigenic phenotype, a transition from the typical elongated shape of human fibroblasts with actin 
organized in fibres to a roundish shape with cortical actin bundles was observed (11). In addition, the invasion of tumorigenic cells relied on ROCK activity, as demonstrated by their decreased invasion capacity upon treatment with a ROCK inhibitor, but not after exposure to an MMP inhibitor (11). At the molecular level, MAT was associated with a reduced expression of the ROCK-1 cellular inhibitor Round (Rnd)3 (also known as RhoE) (11). Exogenous Rnd3 expression led to a reduction in cells' in vitro invasion and in vivo metastasis formation, indicating that Rnd3 levels participate in controlling cellular invasiveness (11).

In the present study, cells undergoing MAT were also demonstrated to be characterized by Snail downregulation, and Snail exogenous expression induced a shift towards an MMP-dependent migration mechanism, further indicating a role for Snail in the modulation of neoplastic cells' movement.

\section{Materials and methods}

Cell lines, cell culture, transfection and plasmids. The cen3tel cellular system has been previously described $(11,12)$. Briefly, it was derived from primary cen 3 fibroblasts by infection with a human telomerase reverse transcriptase (hTERT)-containing retrovirus (13). Telomerase-expressing cells were propagated in culture to population doubling (PD) 1,000 , and a gradual acquisition of the neoplastic phenotype was observed $(11,12)$. In the present study, cen3tel cells at five stages of propagation were used, from cells behaving as normal fibroblasts (early cen3tel cells) to cells at the fifth stage, which were tumorigenic and metastatic in nude mice (phase III tumorigenic cells, PD 1,000). Mid cen3tel cells represented cells at the early phases of transformation, which were anchorage-independent but not tumorigenic. Cells from tumorigenic phases I-III induced tumors in mice with decreasing latency time $(11,12)$. Phase III tumorigenic cells were used as recipient for the transfection with Snail-encoding plasmids.

Cells were grown and transfected as previously described (11). Snail-encoding plasmids, p green fluorescent protein (GFP)-Snail-wild-type (wt) and pGFP-Snail-6SA, were obtained from Addgene Inc. (Cambridge, MA, USA). The plasmid pGPF-Snail-6SA contains the complementary DNA (cDNA) for a Snail protein in which the codons encoding for serine $97,101,108,112,116$ and 120 were mutated to encode for an alanine. These aminoacid changes make the protein more stable, thus preventing its phosphorylation by glycogen synthase kinase 3 beta and subsequent proteasomal degradation (14). Clones isolated after transfection with an empty vector were named $\mathrm{C} 1$ and $\mathrm{C} 2$.

Motility and invasion assays. Cell invasiveness was assayed using modified Boyden chambers (Neuro Probe, Inc., Gaithersburg, MD, USA) with polycarbonate polyvinylpyrrolidone-free nuclepore filters (8-mm pore size) as previously described (11). The ROCK inhibitor Y27632 (cat. \#688000; Calbiochem; Merck Millipore, Darmstadt, Germany) or the MMP inhibitor Ro 28-2653 (kindly provided by Dr H. W. Krell; Roche Diagnostics GmbH, Mannheim, Germany) were used in the invasion assays to inhibit the ameboid or the mesenchymal movement, respectively.
Western blot analysis. Whole-cell lysates were prepared using co-immunoprecipitation (Co-IP) or Laemmli buffer as previously described (11). The anti-Snail antibody (dilution 1:500; clone H-130; Santa Cruz Biotechnology, Inc., Dallas, TX, USA) was used on extracts prepared with the CoIP buffer. The anti-fibronectin antibody (dilution 1:5,000; clone LN-6; Sigma-Aldrich, St.Louis, MO, USA) and the anti-vimentin antibody (dilution 1:2,000; clone S-20; Santa Cruz Biotechnology, Inc.) were used on extracts prepared with the Laemmli buffer.

Microarray analysis. All the details of the microarray analysis and the results obtained are described in Ostano et al (12).

Animal models and ethics statement. To investigate the tumorigenic potential of Snail-expressing clones, $10^{7}$ cells were injected subcutaneously into severe combined immunodeficiency (SCID) mice (Harlan Italy S.r.l., Milan, Italy). For the study, 7-week-old female SCID mice were used, which were housed at $24^{\circ} \mathrm{C}$ in individually ventilated cages with light/dark cycles of $12 \mathrm{~h}$. Mice were provided with sterile food and water ad libitum and manipulated in aseptic conditions. After injection, mice were monitored 2 or 3 times/week to assess tumor appearance and growth. To investigate their metastatic ability, $2 \times 10^{6}$ cells were injected intravenously in SCID mice. Mice were monitored daily and sacrificed at the appearance of distress symptoms. Animals were autopsied, and in order to evaluate metastatic foci, tissues were collected and stored in Bouin's solution.

Procedures involving animals and their care were conducted in conformity with the following laws, regulations and policies governing the care and use of laboratory animals: Italian Governing Law [D.lgs 26/2014, authorisation no. 19/2008-A, issued on March 6, 2008 by the Ministry of Health of Italy (Rome, Italy)]; Mario Negri Institute for Pharmacological Research (Milan, Italy) Institutional Regulations and Policies providing internal authorisation for persons conducting animal experiments (Quality Management System Certificate-UNI EN ISO 9001:2008-Reg. No 8576-A); National Institutes of Health Guide for the Care and Use of Laboratory Animals (2011 edition); European Union directives and guidelines (EEC Council Directive 2010/63/UE); and the Guidelines for the Welfare and Use of Animals in Cancer Research (15).

The animal experiments conducted in the present study have been reviewed and approved by the Animal Care and Use Committee of the Mario Negri Institute for Pharmacological Research, which includes members ad hoc for ethical issues (approval ID Frap1). Animals were housed in the animal care facilities of the Mario Negri Institute for Pharmacological Research, which meet international standards and are regularly checked by a certified veterinarian who is responsible for health monitoring, animal welfare supervision, and experimental protocols and procedures revision.

\section{Results}

Microarray analysis on cen3tel cells representing five different phases of the malignant transformation process exhibited a decrease in Snail expression in cen3tel tumorigenic cells compared with early and mid cen3tel cells (Fig. 1A). The decreased Snail expression with the transformation process was confirmed at the protein level by western blotting (Fig. 1B). 
A

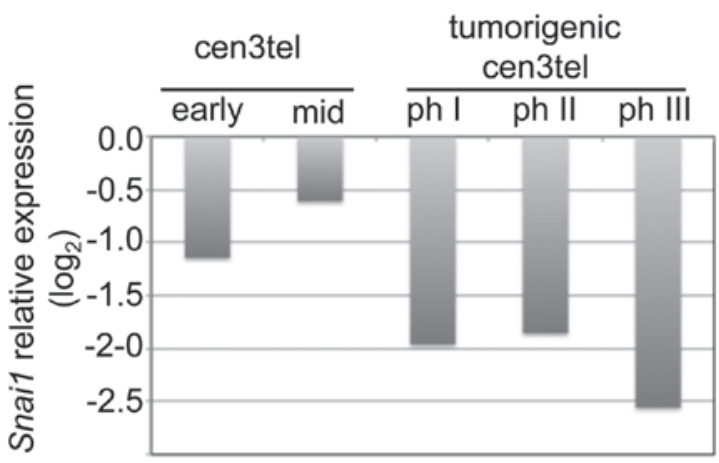

B

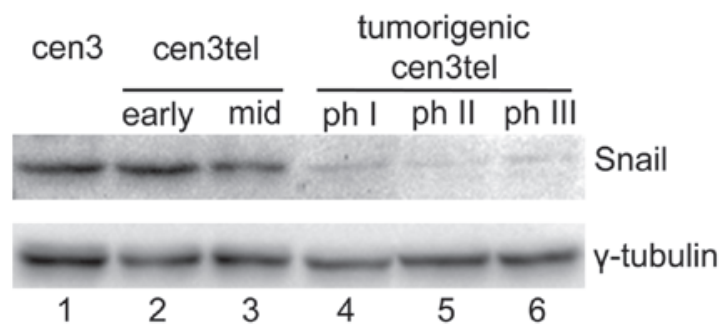

Figure 1. Snal expression in cen3tel cells at different stages of transformation (A) Results of microarray analysis. Snal expression in cen3tel cells is indicated relative to that in cen 3 primary fibroblasts ( $\log _{2}$ of the ratio). (B) Western blot analysis of Snail levels. $\gamma$-tubulin was used as loading control. $\mathrm{Ph}$, phase.

A

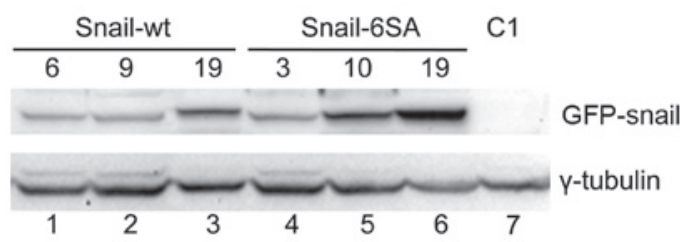

B

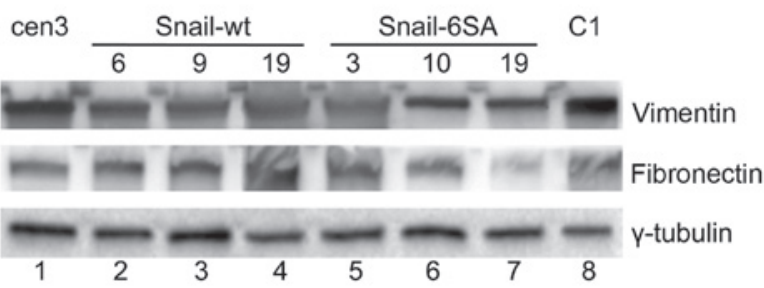

Figure 2. Western blot analysis of the expression of (A) recombinant green fluorescent protein-Snail protein and (B) the mesenchymal markers vimentin and fibronectin in Snail-transfected clones and in mock-transfected cells $(\mathrm{C} 1$, lanes 7A and 8B). $\gamma$-tubulin was used as loading control. WT, wild-type; GFP, green fluorescent protein.

To test whether Snail decreased expression could play a role in modulating the switch from the mesenchymal movement to the amoeboid motility observed in cen3tel tumorigenic cells, Snail was exogenously expressed in phase III tumorigenic cells. For that purpose, cen3tel cells PD 1,000 were transfected either with a plasmid containing the cDNA for the human wt Snail (pGFP-Snail-wt), or a plasmid containing the cDNA for a mutated Snail isoform (pGFP-Snail-6SA), which is more stable. Transfected cells were selected using G418 (Gibco; Thermo Fisher Scientific, Inc., Waltham, MA, USA); resistant clones were isolated

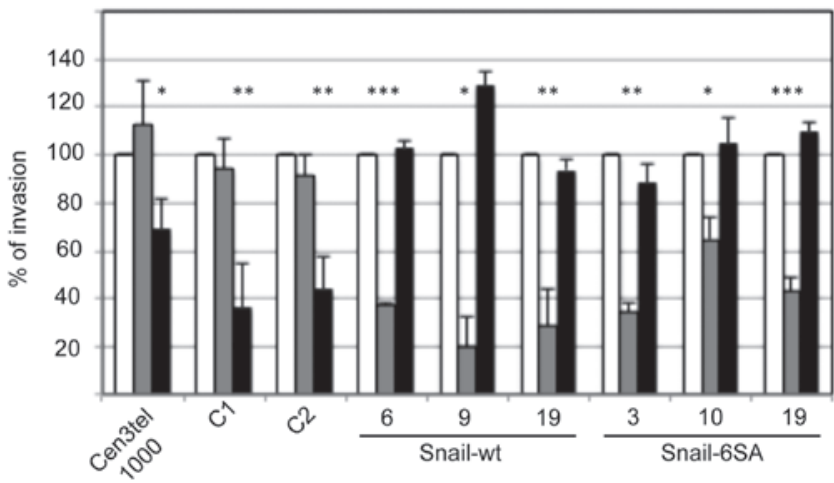

Figure 3. Effect of the Rho-associated kinase inhibitor Y27632 and the matrix metalloproteinase inhibitor Ro 28-2653 on the invasion capacity of phase III tumorigenic cells, mock-transfected cells (C1 and C2 clones) and Snail-expressing clones. Invasion was measured using Boyden chambers. Grey columns, Ro 28-2653 (0.1 mM); black columns, Y27632 (10 mM). Invasion of cells exposed to inhibitors is shown as percentage of invasion in untreated cells (white columns). Data are presented as the mean \pm standard deviation of 2-4 independent experiments. In cen3tel, C1 and C2 cells, the $\mathrm{P}$-value refers to Y27632-treated cells vs. control cells, while in Snail clones, the P-value refers to Ro 28-2653-treated cells vs. control cells $\left({ }^{*} \mathrm{P}<0.05\right.$; $\left.{ }^{* *} \mathrm{P}<0.005 ;{ }^{* * *} \mathrm{P}<0.0005\right)$. WT, wild-type.

and the expression of the GFP-Snail protein was confirmed by fluorescence microscopy (data not shown). Three clones expressing the wt protein (named Snail-wt 6, 9 and 19), and three clones expressing the modified protein (named Snail-6SA 3, 10 and 19) were selected for further investigations. By western blotting with an anti-Snail antibody, Snail expression was analyzed in the clones. As indicated in Fig. 2A, no signal corresponding to the recombinant GFP-Snail protein was observed in the mock-transfected C1 clone (lane 7), as expected, while bands of variable intensities were observed in all other clones. In particular, the highest levels of Snail expression were observed in clones Snail-6SA 10 and 19 (lanes 5 and 6) and in clone Snail-wt 19 (lane 3).

In epithelial tumors undergoing EMT, Snail expression is associated with an increased expression of mesenchymal markers, including vimentin and fibronectin (5). In cen3tel cells, exogenous Snail expression did not positively control the levels of either vimentin or fibronectin (Fig. 2B). Furthermore, it did not induce a change in the organization of the actin cytoskeleton, with actin organized in cortical rings in the clones as in the parental and mock-transfected cells (data not shown).

Whether Snail expression could play a role in determining the type of movement adopted by cen3tel cells was next tested by analyzing the invasion capacity of the Snail-expressing clones in the presence of the ROCK inhibitor Y27632 or the MMP inhibitor Ro 28-2653. As shown in Fig. 3, the two inhibitors had an opposite effect on Snail-expressing clones compared with parental and mock-transfected cells. In fact, while in control and mock-transfected clones ( $\mathrm{C} 1$ and $\mathrm{C} 2)$, invasiveness was decreased by Y27632 but was not affected by Ro 28-2653, as expected, in all Snail-expressing clones, invasiveness was clearly decreased upon exposure to Ro 28-2653 but was not reduced by Y27632, indicating a switch from a ROCK-dependent movement to a protease-dependent motility. 
A

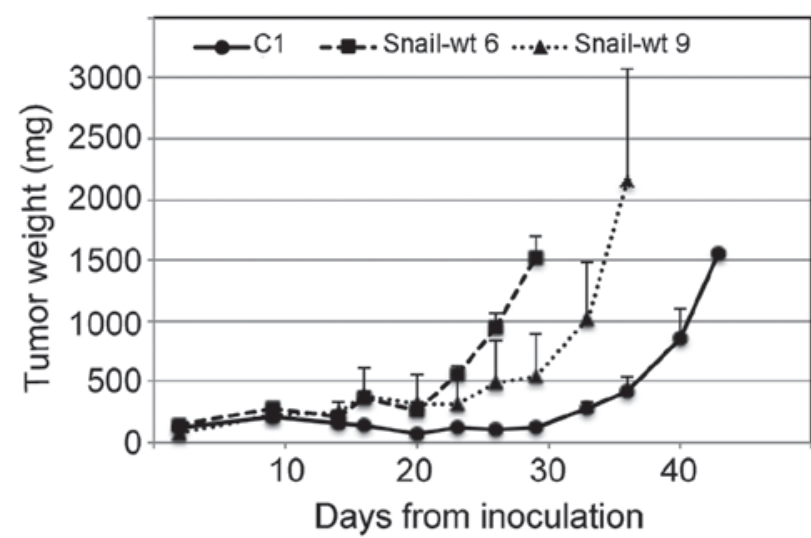

B

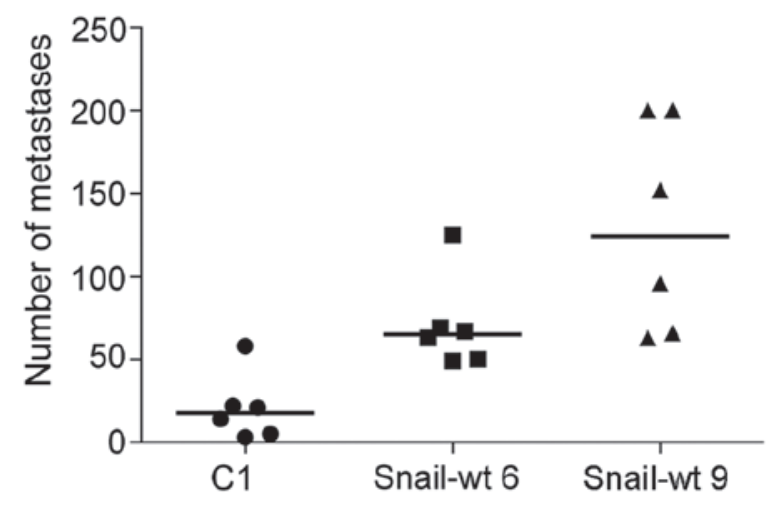

Figure 4. In vivo tumorigenic and metastatic capacity of Snail-expressing clones. (A) Growth curves of tumors obtained in SCID mice (n=3) after subcutaneous inoculation of mock-transfected phase III tumorigenic cen3tel cells (C1) or Snail-expressing clones (Snail-wt 6 and 9). Vertical bars indicate the standard error. (B) Number of lung metastases obtained upon intravenous injection of mock-transfected phase III tumorigenic cen3tel cells (C1) or Snail-expressing clones (Snail-wt 6 and 9) into SCID mice. In two mice inoculated with Snail-wt 9 cells, the precise number of metastases could not be determined due to their large abundance, and was arbitrarily assigned a value of 200. Horizontal bars represent the median number of metastases. WT, wild-type; SCID, severe combined immunodeficiency.

Finally, the in vivo tumorigenicity and metastatic potential of mock-transfected cells and two Snail clones, Snail-wt 6 and 9 , were tested by inoculating cells either subcutaneously or into the tail vein of SCID mice. It was observed that both Snail clones had a faster rate of tumor growth than control cells (Fig. 4A), and generated a greater number of lung metastases compared with mock-transfected cells (Fig. 4B). The size of the metastases was not increased in Snail-expressing clones, with $45 \%$ of metastases being $>1 \mathrm{~mm}$ in mock-transfected cells, compared with only 25 and 14\% in the Snail-wt 6 and 9 clones, respectively.

\section{Discussion}

Cancer cells' capacity to transition from a type of movement to another is a large problem in metastasis fighting (1). In the present study, it has been demonstrated that human fibroblasts undergoing malignant transformation can adopt the amoeboid movement and switch back to an MMP-dependent movement after the induction of Snail expression.

In the cen3tel cellular system, a reduction in Snail expression was observed during in vitro propagation. In cells at the initial phases after hTERT immortalization, Snail levels were similar to those observed in primary fibroblasts. However, when cells became tumorigenic, Snail was downregulated. Despite the low Snail levels, tumorigenic cells neither displayed variations in the expression of mesenchymal markers, including vimentin and fibronectin [lanes 1 and 9 in Fig. 2B and Belgiovine et al (16)], nor started expressing epithelial markers such as cytokeratins (16). In the clones expressing either the wt or the mutated Snail protein, vimentin and fibronectin were expressed at variable levels, but not at levels higher than those in the control cells, indicating that, in cells of mesenchymal origin, the expression of these proteins does not depend on Snail. This supports the hypothesis that Snail is important for the induction of the mesenchymal phenotype, but not for its maintenance (17).
By contrast, Snail levels appeared to be important for the determination of the type of movement used by cancer cells. In fact, exogenous Snail expression in tumorigenic cells induced a switch from a ROCK-dependent movement to a protease-dependent one. In the present study, no differences were observed among clones depending on the levels of Snail or the type of exogenous protein expressed. Exogenous Snail could promote the mesenchymal movement by positively regulating MMP expression and by stimulating invadopodia formation and local matrix degradation through Twist induction (18-20).

In Snail-expressing clones, invasion was insensitive to the ROCK inhibitor Y27632, suggesting that the mesenchymal movement prevails over the amoeboid one. During the transformation process, Snail downregulation may be a pre-requisite for the establishment of the amoeboid movement, which could be induced by low Rnd3 levels and consequent high ROCK activity. This hypothesis is in agreement with the data by Taddei et al (21), indicating that MAT can be engaged by melanoma cells only if EMT is partially suppressed. In tumorigenic cen3tel cells, the mesenchymal phenotype could be partially suppressed upon Snail downregulation. Indeed, in tumorigenic cells, the loss of both the elongated mesenchymal shape and the actin organization in stress fibres was observed.

When injected into the tail vein of immunocompromised mice, Snail-expressing clones exhibited a significantly greater capacity to form lung metastasis than control cells, with an increase in the number, but not in the size, of metastases, suggesting that the protease-dependent movement makes cells more efficient in extravasation than parental cells, which rely on the amoeboid movement. Although it cannot be excluded that the higher growth rate observed in the Snail-expressing tumors compared with that of the tumours induced by mock-transfected cells could facilitate lung colonization, and thus contribute to the higher frequency of metastasis formation, the present results suggest a more aggressive behavior of Snail-expressing cells. 
In conclusion, the present results confirm the role of Snail in directing the mesenchymal movement and the aggressive/metastatic behavior of mesenchymal tumor cells. Furthermore, the present findings highlight the plasticity of tumor cells in adapting their movement in response to changes in gene expression.

\section{References}

1. Sahai E: Mechanisms of cancer cell invasion. Curr Opin Genet Dev 15: 87-96, 2005.

2. Friedl P and Wolf K: Plasticity of cell migration: A multiscale tuning model. J Cell Biol 188: 11-19, 2010.

3. Rath $\mathrm{N}$ and Olson MF: Rho-associated kinases in tumorigenesis: Re-considering ROCK inhibition for cancer therapy. EMBO Rep 13: 900-908, 2012.

4. Lamouille S, Xu J and Derynck R: Molecular mechanisms of epithelial-mesenchymal transition. Nat Rev Mol Cell Biol 15: 178-196, 2014.

5. Kaufhold S and Bonavida B: Central role of Snaill in the regulation of EMT and resistance in cancer: A target for therapeutic intervention. J Exp Clin Cancer Res 33: 62, 2014.

6. Wolf K, Mazo I, Leung H, Engelke K, von Andrian UH, Deryugina EI, Strongin AY, Bröcker EB and Friedl P: Compensation mechanism in tumor cell migration: Mesenchymal-amoeboid transition after blocking of pericellular proteolysis. J Cell Biol 160: 267-277, 2003.

7. Carragher NO, Walker SM, Scott Carragher LA, Harris F, Sawyer TK, Brunton VG, Ozanne BW and Frame MC: Calpain 2 and Src dependence distinguishes mesenchymal and amoeboid modes of tumour cell invasion: A link to integrin function. Oncogene 25: 5726-5740, 2006.

8. Gadea G, de Toledo M, Anguille C and Roux P: Loss of p53 promotes RhoA-ROCK-dependent cell migration and invasion in 3D matrices. J Cell Biol 178: 23-30, 2007.

9. Berton S, Belletti B, Wolf K, Canzonieri V, Lovat F, Vecchione A, Colombatti A, Friedl P and Baldassarre G: The tumor suppressor functions of p27(kip1) include control of mesenchymal/amoeboid transition. Mol Cell Biol 29: 5031-5045, 2009.

10. Parri M, Taddei ML, Bianchini F, Calorini L and Chiarugi $\mathrm{P}$. EphA2 reexpression prompts invasion of melanoma cells shifting from mesenchymal to amoeboid-like motility style. Cancer Res 69: 2072-2081, 2009.
11. Belgiovine C, Frapolli R, Bonezzi K, Chiodi I, Favero F, Mello-Grand M, Dei Tos AP, Giulotto E, Taraboletti G, D'Incalci M and Mondello C: Reduced expression of the ROCK inhibitor Rnd3 is associated with increased invasiveness and metastatic potential in mesenchymal tumor cells. PLoS One 5: e14154, 2010.

12. Ostano P, Bione S, Belgiovine C, Chiodi I, Ghimenti C, Scovassi AI, Chiorino $\mathrm{G}$ and Mondello C: Cross-analysis of gene and miRNA genome-wide expression profiles in human fibroblasts at different stages of transformation. OMICS 16: 24-36, 2012.

13. Mondello C, Chiesa M, Rebuzzini P, Zongaro S, Verri A, Colombo T, Giulotto E, D'incalci M, Franceschi C and Nuzzo F: Karyotype instability and anchorage-independent growth in telomerase-immortalized fibroblasts from two centenarian individuals. Biochem Biophys Res Commun 308: 914-921, 2003.

14. Zhou BP, Deng J, Xia W, Xu J, Li YM, Gunduz M and Hung MC: Dual regulation of Snail by GSK-3beta-mediated phosphorylation in control of epithelial-mesenchymal transition. Nat Cell Biol 6: 931-940, 2004.

15. Workman P, Aboagye EO, Balkwill F, Balmain A, Bruder G, Chaplin DJ, Double JA, Everitt J, Farningham DA, Glennie MJ, et al; Committee of the National Cancer Research Institute: Guidelines for the welfare and use of animals in cancer research. Br J Cancer 102: 1555-1577, 2010.

16. Belgiovine C, Chiodi I and Mondello C: Relocalization of cell adhesion molecules during neoplastic transformation of human fibroblasts. Int J Oncol 39: 1199-1204, 2011.

17. Rowe RG, Li XY, Hu Y, Saunders TL, Virtanen I, Garcia de Herreros A, Becker KF, Ingvarsen S, Engelholm LH, Bommer GT, et al: Mesenchymal cells reactivate Snaill expression to drive three-dimensional invasion programs. J Cell Biol 184: 399-408, 2009.

18. Miyoshi A, Kitajima Y, Kido S, Shimonishi T, Matsuyama S, Kitahara K and Miyazaki K: Snail accelerates cancer invasion by upregulating MMP expression and is associated with poor prognosis of hepatocellular carcinoma. Br J Cancer 92: 252-258, 2005.

19. Ota I, Li XY, Hu Y and Weiss SJ: Induction of a MT1-MMP and MT2-MMP-dependent basement membrane transmigration program in cancer cells by Snail1. Proc Natl Acad Sci USA 106: 20318-20323, 2009.

20. Eckert MA,Lwin TM,Chang AT, Kim J,Danis E,Ohno-Machado L and Yang J: Twist1-induced invadopodia formation promotes tumor metastasis. Cancer Cell 19: 372-386, 2011.

21. Taddei ML, Giannoni E, Morandi A, Ippolito L, Ramazzotti M, Callari M, Gandellini P and Chiarugi P: Mesenchymal to amoeboid transition is associated with stem-like features of melanoma cells. Cell Commun Signal 12: 24, 2014. 